\title{
Benzylation of Toluene over Iron Modified Mesoporous Ceria
}

\author{
K.J. Rose Philo*, S. Sugunan \\ Department of Applied Chemistry, Cochin University of Science and Technology \\ Cochin-682022, Kerala, India
}

Received: 30th June 2012; Revised: 7th November 2012; Accepted: 10th November 2012

\begin{abstract}
Green chemistry has been looked upon as a sustainable science which accomplishes both economical and environmental goals, simultaneously. With this objective, we developed an alternative process to obtain the industrially important benzyl aromatics by benzylation of aromatics using benzyl chloride, catalysed by mesoporous solid acid catalysts. In this work mesoporous ceria is prepared using neutral surfactant which helped the calcination possible at a lower temperature enabling a higher surface area. Mesoporous ceria modified with $\mathrm{Fe}$ can be successfully utilized for the selective benzylation of toluene to more desirable product methyl diphenyl methane with $100 \%$ conversion and selectivity in 2 hours using only $50 \mathrm{mg}$ of the catalyst under milder condition. The reusability, regenerability, high selectivity, $100 \%$ conversion, moderate reaction temperature and absence of solvent, etc. make these catalysts to be used in a truly heterogeneous manner and make the benzylation reaction an environment friendly one. (c) 2012 BCREC UNDIP. All rights reserved.
\end{abstract}

Keywords: Benzylation of aromatics; Green chemistry; Lewis acidity; Mesoporous solid acids

How to Cite: K.J. R. Philo, S. Sugunan. (2012). Benzylation of Toluene over Iron Modified Mesoporous Ceria. Bulletin of Chemical Reaction Engineering \& Catalysis, 7(2): 158-164.

(doi:10.9767/bcrec.7.2.3759.158-164)

Permalink/DOI: http://dx.doi.org/10.9767/bcrec.7.2.3759.158-164

\section{Introduction}

Friedel and Crafts first reported the alkylation of benzene with alkyl chlorides in the presence of aluminium chloride in 1877 [1]. Over the past century, overwhelming number of compounds has been synthesized through the modification of the original approach, a field now commonly referred to as Friedel-Crafts chemistry. Benzyl aromatics are key intermediates in the multifaceted angle of industrial applications. The catalysts used in these reactions are often homogeneous catalyst such as $\mathrm{HF}, \mathrm{H}_{2} \mathrm{SO}_{4}, \mathrm{AlCl}_{3}$ and $\mathrm{BF}_{3}$. Though readily available and inexpensive, these catalysts have numerous drawbacks. Unfortunately, they do not fulfil the current requirements of environmental protection and safety standards. Iron loaded mesoporous materials [2] as catalysts have proved to be highly active and efficient in previous studies. The fundamental concept is to identify new, stable and recyclable catalysts as replacements for the conventional liquid acids, and ultimately develop environmentally safe industrial processes. Herein we report a recyclable, easily separable, ecofriendly and highly effective catalytic system of mesoporous ceria modified with $\mathrm{Fe}$ for the benzylation of toluene with benzyl chloride (BC), a

\footnotetext{
* Corresponding Author. E-mail: marshal_rose@yahoo.com

Tel: +91-484-2543797 Fax: +91-484-2577595
} 
typical example of Friedel-Crafts alkylation.

\section{Materials and Methods}

\subsection{Experimental}

Iron nitrate hexahydrate $\mathrm{Fe}\left(\mathrm{NO}_{3}\right)_{3} \cdot 6 \mathrm{H}_{2} \mathrm{O}$ (Alfa 98.5\%), $\mathrm{Ce}\left(\mathrm{NO}_{3}\right)_{3} .6 \mathrm{H}_{2} \mathrm{O}$ (IRE) and ammonia were used as sources for iron, cerium and alkali respectively. Hexadecyl amine (HDA) (Aldrich $98 \%$ ), was used as surfactant for the synthesis of mesoporous ceria by surfactant method. Mesoporous ceria was synthesized, according to literature procedure [3] and modified with 3 different compositions of $\mathrm{Fe}$ by wet impregnation method.Catalysts were characterized by various analytical and spectroscopic techniques. The acidity measurements of the prepared samples were done by Temperature Programmed Desorption (TPD) of ammonia [4] and the values obtained were tested by vapour phase cumene cracking reaction [5].

\subsection{Synthesis and characterization}

In a typical synthesis procedure mesoporous ceria was prepared according to literature procedure [3]. Since the neutral surfactant inorganic interaction is due to weak hydrogen bonding the removal of most of the surfactant can be done by washing/solvent extraction. The filtered precipitate is dried and then pre-calcined at $250{ }^{\circ} \mathrm{C}$ for 6 hours to make the calcination effective at lower temperature. The sample is then calcined at $350{ }^{\circ} \mathrm{C}$ for $4 \mathrm{~h}$. Mesoporous ceria thus obtained was modified by different weight\% (2, 4, and 10 wt. \%) of iron by wet impregnation method.

\subsubsection{Characterization}

Powder X-ray diffractograms of the materials were recorded on a Rigaku D MAX III VC Nifiltered $\mathrm{Cu}$ Ka radiation, $\lambda=1.5404 \mathrm{~A}^{\circ} 2 \theta$ range $10-80^{\circ}$ at a speed of $1 \% \mathrm{~min}$. Low angle XRD was also done to confirm the mesoscopic nature of the sample. $\mathrm{N}_{2}$ adsorption-desorption isotherms, pore size distributions as well as the textural properties of the materials were determined at $-196{ }^{\circ} \mathrm{C}$ by a Micromeritics Tristar 3000 surface area and porosity analyser. Prior to the measurements the samples were degassed for $1 \mathrm{~h}$ at $90{ }^{\circ} \mathrm{C}$ followed by $200^{\circ} \mathrm{C}$ overnight. Pore size distribution and average pore size of the samples were obtained from the adsorption branch of the isotherms using the Barrnet-Joyner-Halenda (BJH) method. FTIR spectra of the solid samples were taken in the range of $4000-400 \mathrm{~cm}^{-1}$ on a Shimadzu FTIR 8201 instrument by diffuse reflectance scanning disc technique. Diffuse reflectance UV-Vis spectra were recorded in the range $200-800 \mathrm{~nm}$ with a Shimadzu UV-2101 PC spectrometer equipped with a diffuse reflectance attachment, using $\mathrm{BaSO}_{4}$ as the reference. SEM analysis of the samples was done using JEOL JSM-840 A (Oxford make) model16211 scanning electron microscope analyzer with a resolution of $13 \mathrm{eV}$. The HR-TEM of the sample was carried out in ultrahigh resolution analytical electron microscope JEOL3010. Perkin Elmer TG analyzer instrument was used for carrying out thermo gravimetric studies. About 20 $\mathrm{mg}$ of the sample was used at a heating rate of 20 ${ }^{0} \mathrm{C}$ per min in nitrogen atmosphere. The TG data were computer processed to get thermograms. Elemental composition was confirmed by ICP-AES.

The acidity measurements of the prepared samples were done by Temperature Programmed Desorption (TPD) using ammonia as probe molecule by desorbing ammonia at various temperature after adsorption on the catalyst at room temperature. The values obtained were tested by vapour phase cumene cracking reaction . Vapour phase cumene cracking reaction is a test reaction for identifying the Lewis to Bronsted acid ratio of a catalyst. The major reactions taking place during the cracking of cumene are dealkylation to give benzene and propene over Bronsted acid sites (BAS) and dehydrogenation to give a-methyl styrene over Lewis acid sites (LAS). A comparison of the amount of dealkylated products and a-methyl styrene gives an idea about the (BAS) and (LAS) possessed by the catalyst .

\subsubsection{Benzylation Reaction}

To study the activity of the catalysts in organic reactions, benzylation of toluene was done using benzyl chloride in the presence of the prepared catalysts. The benzylation reaction was carried out in a double necked round bottom flask fitted with a reflux condenser. In a typical run, appropriate amounts of benzyl chloride and the catalyst were allowed to react at specified temperatures under magnetic stirring for 25 minutes and then toluene was added to the reacting mixture. The reaction mixtures were analysed periodically using $8610 \mathrm{GC}$ equipped with SE-30 column and FID detector. As toluene was taken in excess, the yield of the reaction was expressed as the total percentage of benzyl chloride transformed. The product formed was identified as o/p methyl diphenyl methane by GCMS and also by comparison with the peaks obtained for the standard.

\section{Results and Discussion}

The crystalline phases were identified by 
comparison with standard JCPDS (Joint Committee on Powder Diffraction Standards) data file [6]. Wide angle XRD analysis clearly shows peaks corresponding to the crystalline cubic fluorite ceria phase (PDF-ICDD34-0394). With loading of $\mathrm{Fe}$ no additional iron oxide phase was formed up to a $10 \mathrm{wt} \%$ Fe loading (Figure 1). The characteristic peak of crystalline iron oxide is absent in lower loading. This points to the fact that the iron species are highly dispersed or exist as microcrystalline material below the XRD detection limit. A peak at low-angle region confirms the mesoporous nature of the sample prepared (Figure 1a). The sample exhibits $\mathrm{N}_{2}$ adsorption-desorption of Type IV isotherm with steps between partial pressures $\mathrm{P} / \mathrm{Po}$ of 0.3 to 0.8 , and a hysteresis loop, due to capillary condensation in the mesoporous

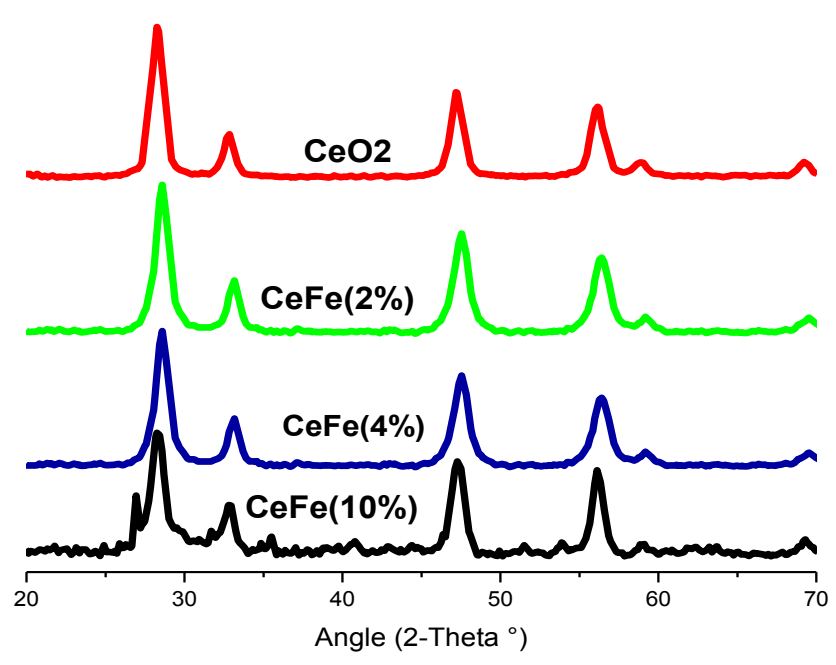

Figure 1a: Wide-angle XRD pattern of mesoporous ceria and ceria modified with $\mathrm{Fe}$

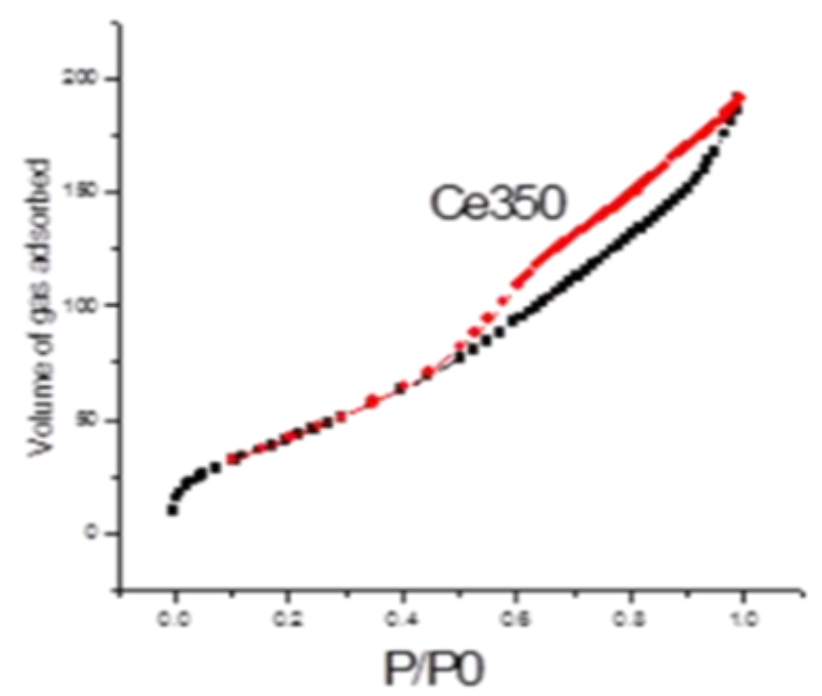

Figure 2: Nitrogen sorption isotherm of mesoporous ceria channels and/or cages (Figures 2 and 3). Figure 2a shows the pore size distribution of the sample. Narrow BJH pore size distribution suggests an open pore channel. It also shows a regular mesopore structure. The H3 hysteresis loop is characteristic of mesoporous materials with narrow slit-like pores.

The pore volume, pore diameter \& surface area calculated by desorption studies are given in (Table 1). From (Table 1) it is evident that surface area decreases with metal loading. Crystallite size and lattice parameters are calculated from XRD data (Table 2) using Scherrer equation. crystallite sizes obtained shows a decrease with metal incorporation. It is clear that with incorporation of heteroatom in the framework of ceria, a slight

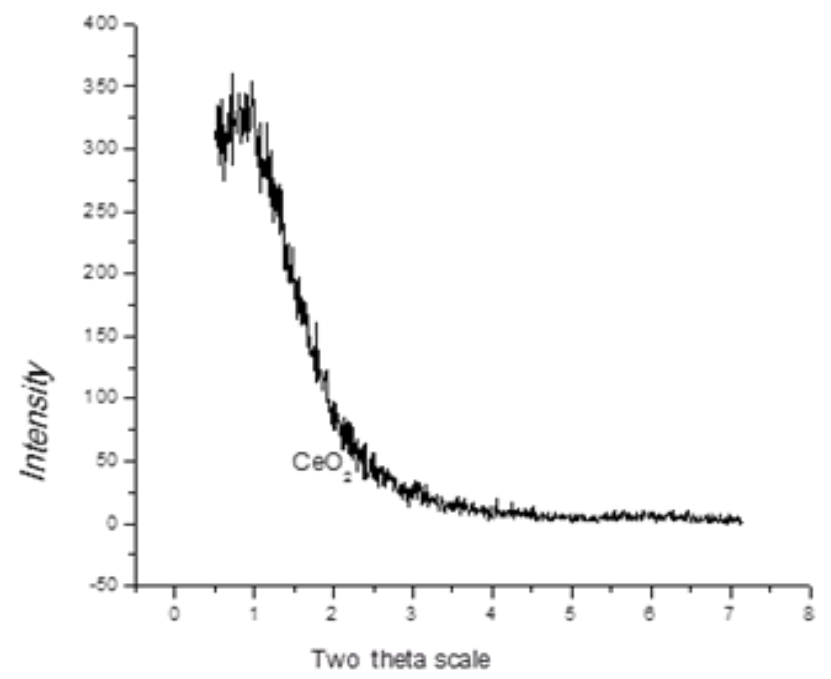

Figure 1b: Low-angle XRD pattern of mesoporous ceria

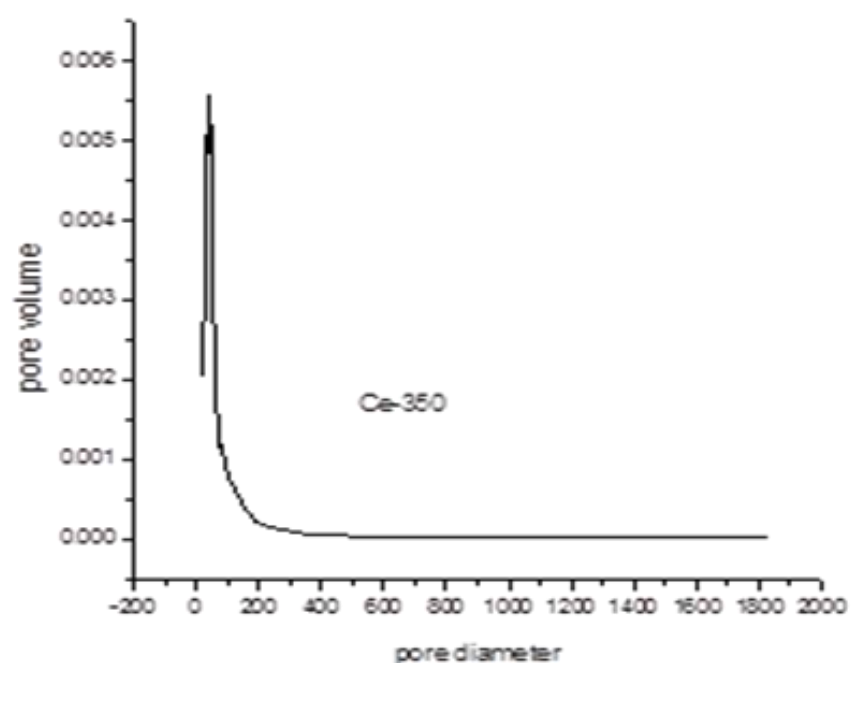

Figure 2a: Pore size distribution curve 
Table 1: Surface area, Pore measurement

\begin{tabular}{lccc}
\hline sample & $\begin{array}{c}\text { BET S.A } \\
\left(\mathrm{m}^{2} \mathrm{~g}^{-1}\right)\end{array}$ & $\begin{array}{c}\text { Average } \\
\text { Pore Di- } \\
\text { ameter } \\
(\mathrm{nm})\end{array}$ & $\begin{array}{c}\text { Cumula- } \\
\text { tive Pore } \\
\text { Volume } \\
\left(\mathrm{cm}^{3} \mathrm{~g}^{-1}\right)\end{array}$ \\
\hline $\mathrm{Ce} 350$ & 164 & 4.19 & 0.29 \\
$\mathrm{CeFe}(2 \%)$ & 100 & 4.5 & 0.18 \\
$\mathrm{CeFe}(4 \%)$ & 99 & 5.2 & 0.17 \\
$\mathrm{CeFe}(10 \%)$ & 52.6 & 4.6 & 0.12 \\
\hline
\end{tabular}
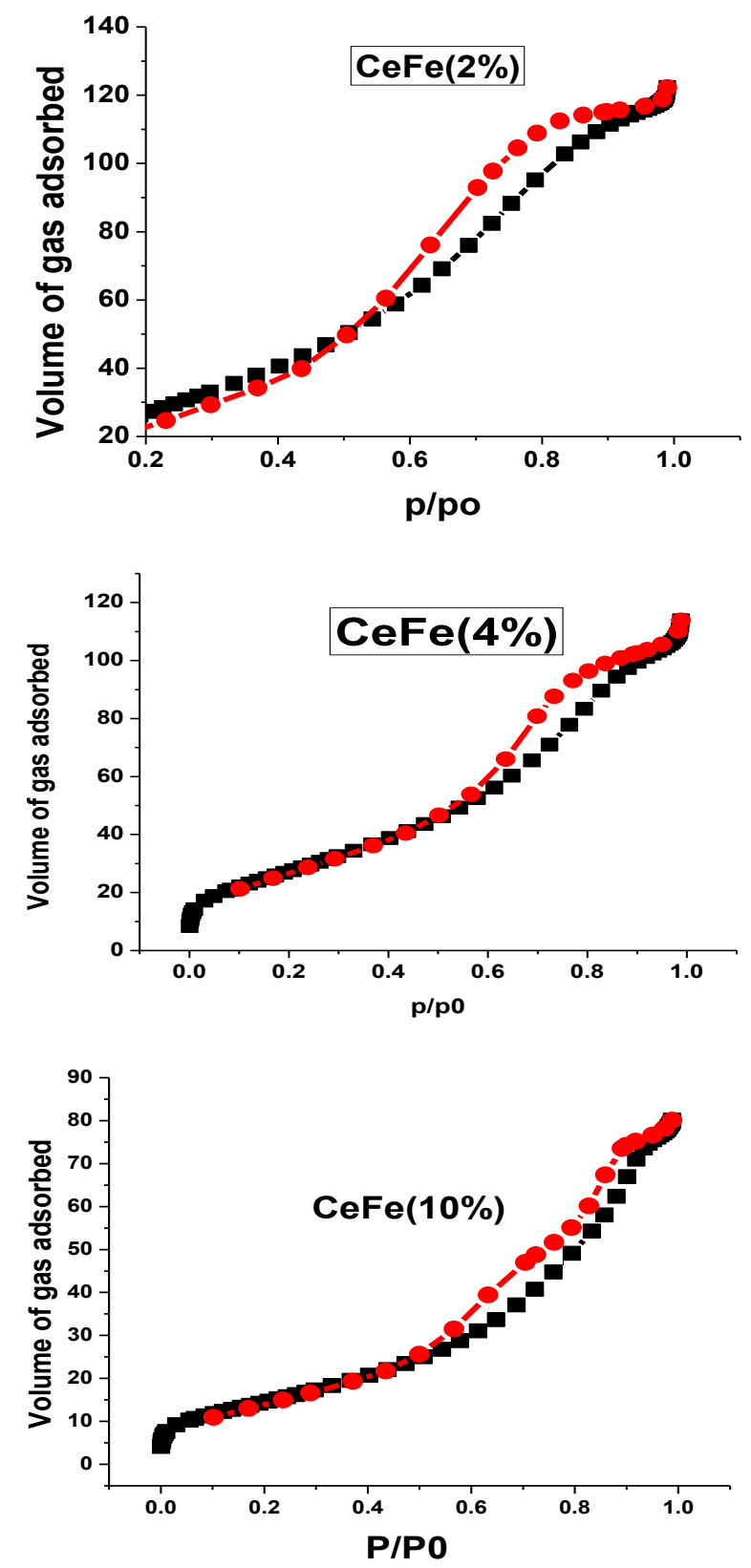

Figure 3: Adsorption isotherms of ceria modified with different metals
Table 2: XRD data

\begin{tabular}{lccc}
\hline Sample & $\begin{array}{c}\text { Average } \\
\text { crystal- } \\
\text { lite size } \\
(\mathrm{nm})\end{array}$ & $\begin{array}{c}\text { Lattice } \\
\text { parameter } \\
(\mathrm{nm})\end{array}$ & $\begin{array}{c}\text { d-spacing } \\
\text { (nm) }\end{array}$ \\
\hline $\mathrm{Ce} 350$ & 12.8 & 0.545 & 3.15 \\
$\mathrm{CeFe}(4 \%)$ & 10.26 & 5.39 & 3.12 \\
$\mathrm{CeFe}(10 \%)$ & 10.66 & 5.44 & 3.15 \\
\hline
\end{tabular}

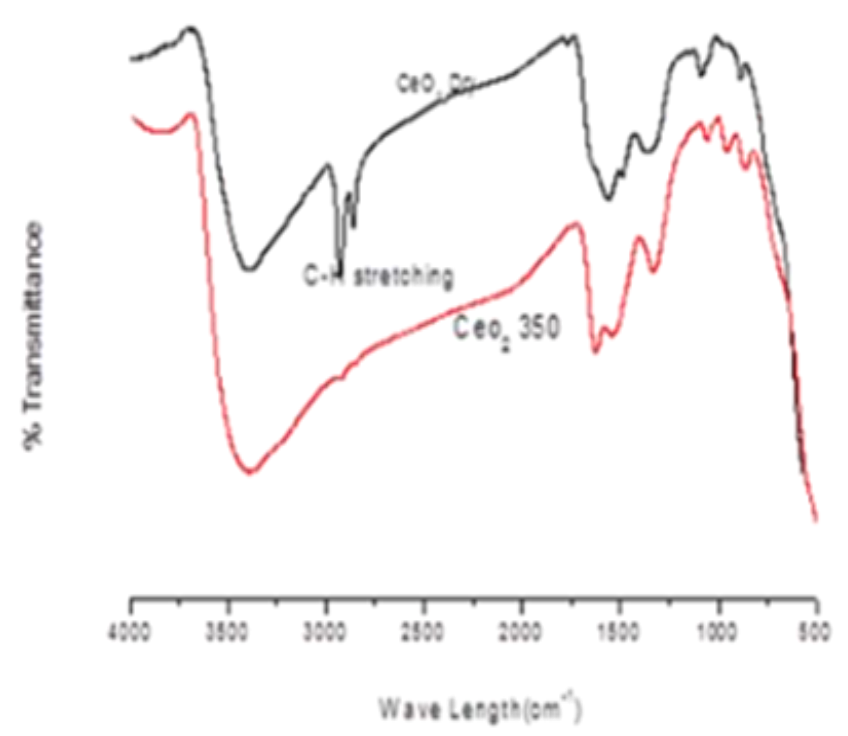

Figure 4a: FT-IR spectrum of sample before and after calcination

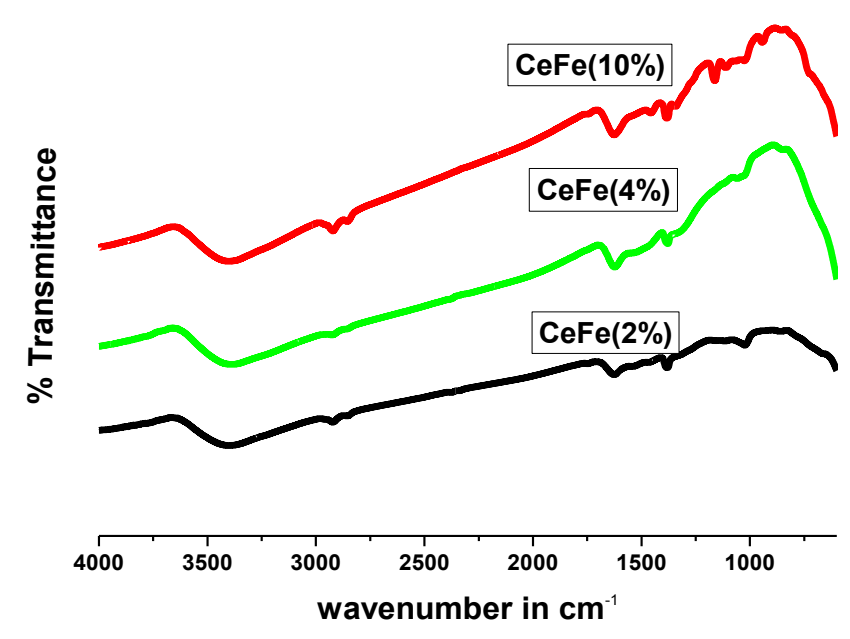

Figure 4b: FT-IR spectra of Fe modified samples 
decrease in the $\mathrm{d}_{111}$ spacing is observed, indicating the presence of the heteroatom within the framework.

In FT-IR spectrum (Figures 4a, 4b) of the calcined materials, bands related to the surfactant are removed which shows the successful removal of surfactant at a lower calcination temperature (350 $\left.{ }^{\circ} \mathrm{C}\right)$ which is effective for a high surface area for the calcined sample free of surfactant. No peaks corresponding to the $\mathrm{C}-\mathrm{H}$ bands are seen in calcined sample. In the spectrum, peak at $3400 \mathrm{~cm}^{-}$ ${ }^{1}$ corresponds to the $\mathrm{OH}$ stretching vibration, the peak at $1630 \mathrm{~cm}^{-1}$ corresponds to the $\mathrm{H}_{2} \mathrm{O}$ bending vibration, Peak at $1380 \mathrm{~cm}^{-1}$ to $\mathrm{Ce}-\mathrm{OH}$ stretching vibration, Peaks $1075 \mathrm{~cm}^{-1}$ and $860 \mathrm{~cm}^{-1}$, are attributed to the stretching and bending vibrations of M-O-M bonding $500 \mathrm{~cm}^{-1}$ corresponds to the $\mathrm{CeO}_{2}$ stretching [7].

HR-TEM shows the formation of uniform crystallites of few nanometres in size. Calcined

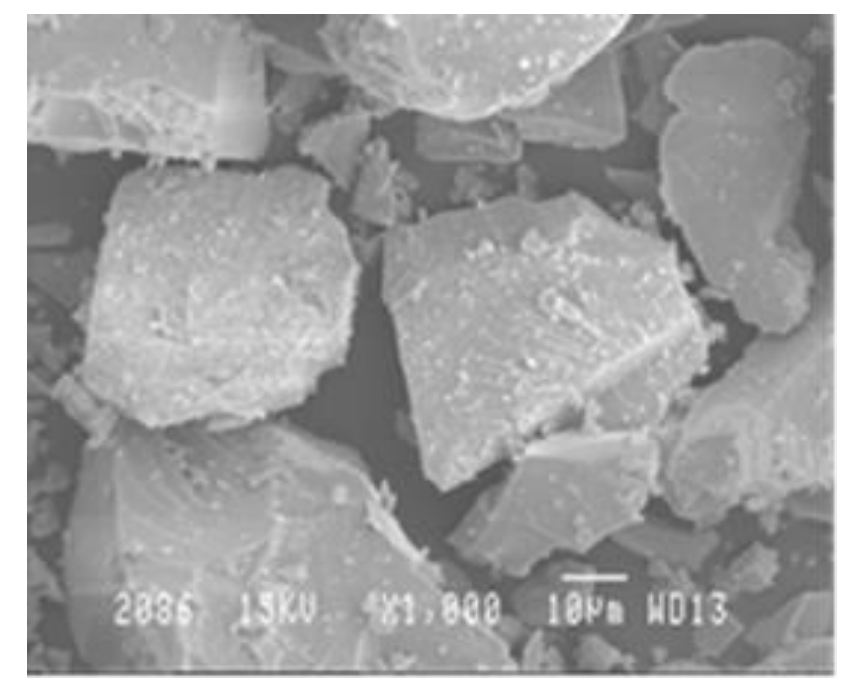

Figure 5a: SEM of mesoporous ceria

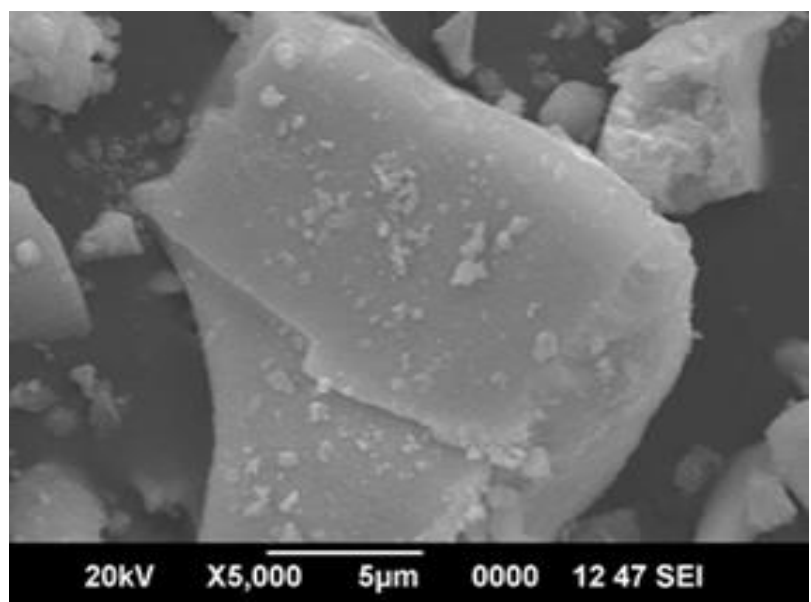

Figure 5b: Mesoporous ceria modified with $2 \% \mathrm{Fe}$ sample consists of aggregated small crystallites of a few nanometres and very homogeneous in size. The lattice fringes visible in the HR-TEM image displayed in Figure 6a are indicative of the high crystallinity of these particles. The selected area electron diffraction (SAED) pattern confirms the formation of the (111) surface plane. Ceria crystallizes in a cubic fluorite structure and exposes the thermodynamically most stable (111) surface. This surface is the oxygen termination of stoichiometric $\mathrm{O}-\mathrm{Ce}-\mathrm{O}$ trilayers stacked along the (111) direction and also represents the major fraction of the catalytic surface in the ceria nanocrystallites. DRS spectrum clearly shows a single band arising from ceria species around $270 \mathrm{~nm}$ which shows that the $\mathrm{Ce}^{4+}$ species are in the tetra co-coordinated environment.

The acidities of the samples measured by TPD of ammonia indicate an increase in the total acidity

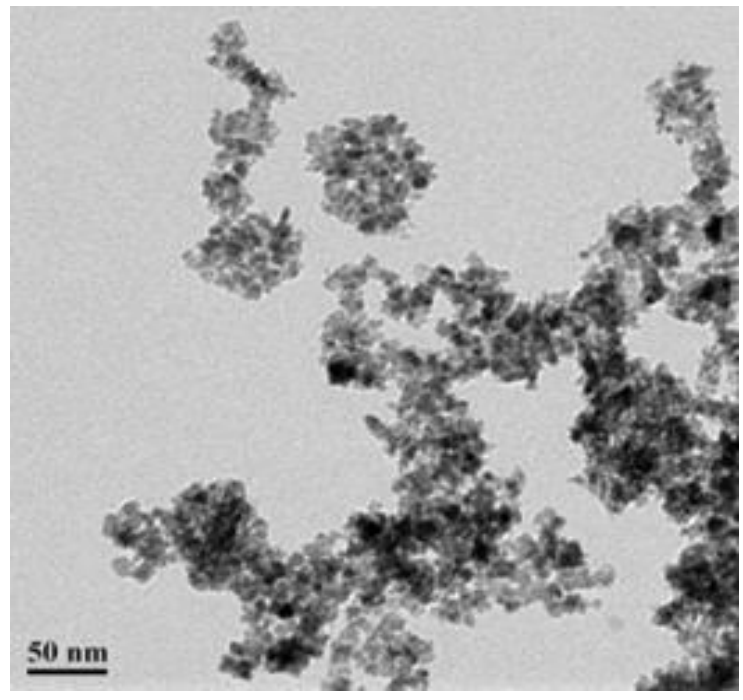

Figure 6a: TEM image of mesoporous ceria

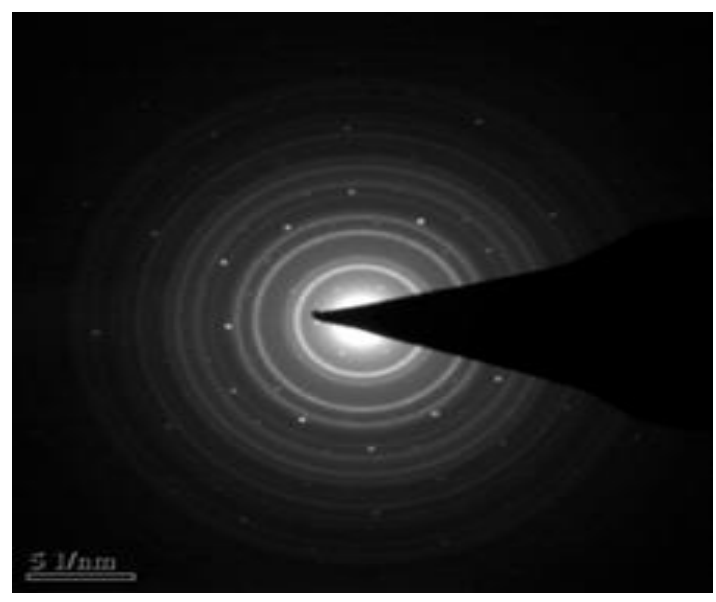

Figure 6b: SAED pattern of mesoporous ceria 
Table 3: Acidity values obtained from TPD of ammonia, \& vapour phase cumene cracking reaction

\begin{tabular}{|c|c|c|c|c|c|c|}
\hline \multirow{2}{*}{ sample } & \multicolumn{4}{|c|}{$\operatorname{Acidity}\left(\mathrm{mmolg}^{-1}\right)^{a}$} & \multirow{2}{*}{$\begin{array}{l}\text { LAS/BAS } \\
\text { acidity }^{b}\end{array}$} & \multirow{2}{*}{$\begin{array}{c}\text { Cumene Convn } \\
(\%)^{c}\end{array}$} \\
\hline & weak & Medium & strong & Total & & \\
\hline $\mathrm{Ce}$ & 0.10 & 0.02 & nil & 0.12 & 1.3 & 3.5 \\
\hline $\mathrm{CeFe}(2 \%)$ & 0.09 & 0.07 & 0.01 & 0.17 & 2.8 & 18.5 \\
\hline $\mathrm{CeFe}(4 \%)$ & 0.11 & 0.02 & 0.03 & 0.16 & 1.7 & 17 \\
\hline $\mathrm{CeFe}(10 \%)$ & 0.09 & 0.02 & 0.02 & 0.13 & 1.3 & 14.2 \\
\hline
\end{tabular}

a The acidity values equivalent to desorbed ammonia'

$b$ The ratio of Lewis to Bronsted acidity obtained from the cumene cracking test reaction foracidity;

c In vapour phase cumene cracking test reaction.

Table 4: Comparison of catalytic activity

\begin{tabular}{ccccc}
\hline sample & $\begin{array}{c}\text { Conversion } \\
(\%)\end{array}$ & $\begin{array}{c}\text { Product }(\% \\
\text { selectivity })\end{array}$ & TON $^{\mathrm{a}}$ & $\mathrm{TOF}^{\mathrm{l}} \mathrm{h}^{\mathrm{b}}$ \\
$\mathrm{Ce}$ & 4.3 & 100 & - & - \\
$\mathrm{CeFe}(2 \%)$ & 100 & 100 & 47800 & 23900 \\
$\mathrm{CeFe}(4 \%)$ & 100 & 100 & 24000 & 12000 \\
$\mathrm{CeFe}(10 \%)$ & 100 & 100 & 9600 & 4800 \\
\hline
\end{tabular}

a-Turnover Number $=($ moles of BC converted $/$ moles of metal loaded in the catalyst) $x 100$; b-Turn over Frequency=TON $/$ time

(Reaction time: 2 hours, weight of catalyst: 0.05g, Temperature $-80^{\circ} \mathrm{C}$, Substrate to $\mathrm{BC}$ ratio-5:1)

with the Fe loading in the sample (Table 3). The table indicates that pure ceria possesses low surface acidity. Upon modification with $\mathrm{Fe}$, there is enhancement in the amount of weak, medium and strong acid sites. But as the metal concentration increases though there is a change in the total acidity, there is no steady increase in the acidity with increase in the concentration of the metals.

Vapour phase cumene cracking reaction is a model reaction for identifying the Lewis to Bronsted acid ratio of a catalyst. The catalytic activity of solid acid catalysts is not only related to the surface concentration of acid sites, but also to their nature, that is being Lewis or Brönsted sites. Good correlation is observed for total acidity with cumene conversion and Lewis to Brönsted acidity
Table 5: Results of reusability study

\begin{tabular}{rcc}
\hline Catalyst & No. of Cycle & \% Conversion \\
\hline \multirow{2}{*}{$\mathrm{CeFe}(10 \%)}$, & I & 100 \\
& II & 100 \\
& III & 98 \\
\hline
\end{tabular}

Reaction conditions: Catalyst: $50 \mathrm{mg} \mathrm{CeFe}$ (10\%), Temperature: $80^{\circ}$ C,Ratio: 5:1, Time: 2 hours

ratio with strong acidity obtained from TPD of ammonia. Acidity studies confirm the enhancement of surface acidity in particular strong acidity, upon modification with iron.

Comparison of catalytic activities of different $\mathrm{Fe}$ loaded catalysts towards the benzylation reaction are given in Table [4]. Reusability study (Table 5) indicates the true heterogeneous nature of the reaction. Pure ceria gave low conversion than $\mathrm{Fe}$ modified samples. The metal modification improves the \% conversion to a great extent. The increase in \% conversion and activity may be attributed to the increase in the acid sites upon metal modification. The conversion is found to be correlated with the total acidity of the samples. The high activity of $\mathrm{Fe}$ systems could not be explained on the basis of their acidity alone. This large hike in the conversion points to the fact that benzylation activity of iron loaded systems cannot be explained on the basis of usual carbocation mechanism for alkylation reaction. It is reported that catalysts containing reducible cations like $\mathrm{Fe}^{3+}, \mathrm{Cu}{ }^{2+}$ etc. exhibit high alkylation activity 
regardless their Lewis acidity [8]. When a redox mechanism is operating there will be homolytic fission of carbon- chlorine bond of the alkylating agent (BC). The radicals so formed are powerful reductants, which would be readily oxidised to carbocations in the presence of reducible metallic $\mathrm{Fe}^{3+}$. During the optimization of parameters for reaction, it was observed that, when the temperature was below $80^{\circ} \mathrm{C}$ there was no reaction taking place. It may be assumed that below $80{ }^{\circ} \mathrm{C}$, the energy for the homolytic rupture of carbonchlorine bond may not be attained and therefore no reaction occurs below this temperature [9]. The effect of temperature and an induction period for the reaction suggests a free radical mechanism for benzylation using benzyl chloride with Fe modified catalyst. Hence catalytic activity of $\mathrm{Fe}$ modified systems compared to the mesoporous ceria can be attributed to the increased acidity and also redox property of $\mathrm{Fe}$.

\section{Conclusion}

Neutral surfactant route using Hexa Decyl Amine (HDA) as surfactant is effective for the preparation of mesoporous ceria with high surface area and pore volume at a lower calcination temperature. Mesoporous ceria modified with $\mathrm{Fe}$ can be successfully utilized for the selective benzylation of toluene to more desirable product methyl diphenyl methane with $100 \%$ conversion and selectivity in 2 hours with low catalyst loading under milder reaction conditions.

The catalysts are regenerable and can be recycled upto third cycle without loss of activity. This reusability, regenerability, high selectivity, $100 \%$ conversion, moderate reaction temperature and absence of solvent etc. make these catalyst to be used in a truly heterogeneous manner and makes the benzylation reaction an environment friendly one. Hence this solid acid catalyst can be used in efficient green chemical process for the manufacture of industrially important benzylated aromatic compounds.

\section{Acknowledgement}

The financial support from University Grants Commission (India) to Rose Philo K.J to undergo Faculty Improvement Programme of UGC XI plan is gratefully acknowledged.

\section{References}

[1] Friedel, C., and Crafts, J.M., (1877). Compt. Rend. Acad. Sci, Paris 84: 1292.

[2] Rupa, V., Palanichami, A. (2008). Phenol Hydroxylation Using Fe/Al-MCM-41 Catalysts. Catal. Lett.120: 56-64.

[3] Tanev, P. T., Pinnavaia, T. J., (1995), A Neutral Templating Route to Mesoporous Molecular Sieves. Science (New York, NY) 267 (5199): 865- 867.

[4] Tanabe, K., Misono, M., Ono, Y., (1989), Solid Acids and Bases. Elsevier Science Publishers, Tokyo-Amsterdam, p.252.

[5] Bradley, S.M., and Kydd, R.A., (1993), Chromium pillared montmorillonites: acidity and reactivity for cumene conversion. $J$ Catal. 141: 239-249.

[6] Brauner, S., Emmette, P.H., Teller, E., (1938) Adsorption of Gases in Multimolecular Layers. J. Am. Chem. Soc., 60 (2): 309-319.

[7] Tillirou, A.A., and Theocharis, C.R., (2008). Synthesis and Characterization of Mesoporous Cerium Oxide Prepared Using an Organic Base and a Templating Agent. Adsorption Science \& Technology 26: 687-692.

[8] Despande, A. B., Bajpai, A. R., Samant, S.D., (2001). The enhanced activity of $\mathrm{Sb}$ after supporting on K10 in the benzylation of benzene using benzyl chloride and benzyl alcohol. Appl. Catal. A: Gen., 209: 229-236.

[9] He, N., Bao, S., Xu, Q., (1998). Fe-containing mesoporous molecular sieves materials: very active Friedel-Crafts alkylation catalysts. Appl. Catal. A: Gen., 169: 29-36. 